\title{
ON SOLUTIONS WITH POLYNOMIAL GROWTH TO AN AUTONOMOUS NONLINEAR ELLIPTIC PROBLEM
}

\author{
KELEI WANG AND JUNCHENG WEI
}

\begin{abstract}
We study the following nonlinear elliptic problem

$$
-\Delta u=F^{\prime}(u) \text { in } \mathbb{R}^{n}
$$

where $F(u)$ is a periodic function. Moser (1986) showed that for any minimal and nonself-intersecting solution, there exist $\alpha \in \mathbb{R}^{n}$ and $C>0$ such that

$$
|u-\alpha \cdot x| \leq C .
$$

He also showed the existence of solutions with any prescribed $\alpha \in \mathbb{R}^{n}$. In this note, we first prove that any solution satisfying $\left(^{*}\right)$ with nonzero vector $\alpha$ must be one dimensional. Then we show that in $\mathbb{R}^{2}$, for any positive integer $d \geq 1$ there exists a solution with polynomial growth $|x|^{d}$.
\end{abstract}

\section{Introduction and Main Results}

In search of analogue of Aubry-Mather theory for quasilinear partial differential equations in $\mathbb{R}^{n}$, Moser [6] studied the following equation

$$
\sum_{i=1}^{n} \frac{\partial}{\partial x_{i}} \mathcal{F}_{p_{i}}(x, u, D u)-\mathcal{F}_{u}(x, u, D u)=0
$$

which is the Euler-Lagrangian equation for the functional

$$
\int_{\mathbb{R}^{n}} \mathcal{F}(x, u, D u) d x
$$

where $\mathcal{F}$ is 1 -periodic in all variables $x_{1}, \ldots, x_{n}$ and $u$, elliptic and of quadratic growth in $p=D u$.

A solution $u(x)$ of (1.1) is called minimal if

$$
\int_{\mathbb{R}^{n}}[\mathcal{F}(x, u+\varphi, D u+D \varphi) d x-\mathcal{F}(x, u, D u)] d x \geq 0, \forall \varphi \in C_{0}^{\infty}\left(\mathbb{R}^{n}\right) .
$$

A solution of (1.1) is said to be without self intersections or WSI if (i) for each $j \in \mathbb{Z}^{n}$ and $j_{n+1} \in \mathbb{Z}, u(x+j)-u(x)-j_{n+1}$ does not change sign for $x \in \mathbb{R}^{n}$, or (ii) for some $j \in \mathbb{Z}^{n}$ and $j_{n+1} \in \mathbb{Z}, u(x+j) \equiv u(x)+j_{n+1}$.

For minimal and WSI solutions to (1.1), Moser [6] showed: (1) There exists a unique vector $\alpha \in \mathbb{R}^{n}$, the so-called rotation vector and a constant $C$, such that

$$
|u(x)-\alpha \cdot x| \leq C, \forall x \in \mathbb{R}^{n} .
$$

1991 Mathematics Subject Classification. 35J25, 35B45, 35B33.

Key words and phrases. minimal solutions, Aubry-Mather theory, harmonic polynomials. 
(2) Conversely, for every vector $\alpha \in \mathbb{R}^{n}$ there exists a minimal solution $u$ with rotation vector $\alpha$ and a constant $C$ and satisfying (1.4).

Moser's paper [6] has received lots of attention in the literature. Among many results, we mention that Bangert [2] showed the existence of heteroclinic states under some gap conditions, and Rabinowitz and Stredulinsky [8, 9] developed variational gluing methods for mixed states of Allen-Cahn type equations. (See also [10] for nonautonomous case.) There is also a strong connection between Moser's problem and De Giorgi's conjecture. See Farina and Valdinoci [5]. For the latest developments, we refer to the survey paper by Rabinowitz [7] and the references therein.

In this note, we consider the autonomous Moser's problem, namely we study the following problem

$$
-\Delta u=F^{\prime}(u) \text { in } \mathbb{R}^{n}
$$

where $F(u)$ is a smooth periodic function. A typical example is the so-called sineGordon nonlinearity $F(u)=1-\cos (u)$.

Our first result is a classification theorem on solutions to (1.5) satisfying (1.4).

Theorem 1.1. Let $u \in C^{2}\left(\mathbb{R}^{n}\right)$ be a solution of (1.5). Assume that there exist a nonzero vector $\alpha \in \mathbb{R}^{n}$ and a constant $C>0$ such that

$$
|u(x)-\alpha \cdot x| \leq C \text { for } \forall x \in \mathbb{R}^{n} .
$$

Then there is a function $v \in C^{2}\left(\mathbb{R}^{n}\right)$ such that $u(x)=v(\alpha \cdot x)$.

In the above theorem, $\alpha \neq 0$ is necessary. In fact for Allen-Cahn or Sine-Gordon equations, there are bounded solutions with multiple transitions $([1,3,3,4])$. Theorem 1.1 also holds when $-\Delta u=f(u)$ where $f$ is periodic. Note that it can be directly shown that one dimensional solutions satisfying (1.6) have no self-intersection.

Theorem 1.1 has been proved by Farina and Valdinoci [5] under the minimality condition. Here we have removed the minimality assumption. Theorem 1.1 shows that unbounded solutions to (1.5) with linear growth are all one dimensional. Notice that $\alpha \cdot x$ is the simplest nonconstant harmonic function in $\mathbb{R}^{n}$. Based on this, J. Byeon and P. Rabinowitz 1 asked

Question: given any harmonic function, $w$, on $\mathbb{R}^{n}$, is there a solution, $u$, of (1.1) with $\|u-w\|_{L^{\infty}\left(\mathbb{R}^{n}\right)}$ bounded?

The following theorem answers the question partially.

Theorem 1.2. Let $n=2$ and $d \geq 2$. Assume that $F(u)$ is even. Let $\varphi(x, y)$ be the real part of the harmonic polynomial $z^{d}$. (Here $z=x+i y$.) Then there exists a solution to (1.5), enjoying the same symmetry as $\varphi(x, y)$ and satisfying

$$
|u(x, y)-\varphi(x, y)| \leq C(1+|z|)^{\frac{3}{2}} .
$$

Furthermore, for $d \geq 3$ we also have the following improved upper bound:

$$
|u(x, y)-\varphi(x, y)| \leq C(1+|z|)^{2-\frac{d}{2}} .
$$

\footnotetext{
${ }^{1}$ Private discussion
} 
Remark 1.3. If $d \geq 4$, then $\frac{d}{2} \geq 2$. Thus for $d \geq 4$, we answered ByeonRabinowitz's question affirmatively, in the autonomous setting (1.5). Note also that for $d>4$, we have better decay estimates. The key to obtain (1.8) is some oscillatory integral estimate (see (4.6) below). For $d=2$ or 3 , this estimate is not sufficient. We believe that the $L^{\infty}$ bound should also hold for $d=2,3$.

Remark 1.4. Another interesting question is whether or not the evenness condition is necessary.

In the rest of the paper, we prove Theorem 1.1 in Section 2, the estimate (1.7) of Theorem 1.2 in Section 3 and the better estimate (1.8) of Theorem 1.2 in Section 4 respectively.

Acknowledgment. The second author thanks Professors J. Byeon and P. Rabinowitz for suggesting the problem and nice discussions.

\section{Proof of Theorem 1.1}

In this section, we prove Theorem 1.1 by the method of moving planes.

Without loss of generality, assume that $|\alpha|=1$ and $\alpha$ is the $x_{n}$ direction. We use the notation that $x=\left(x^{\prime}, x_{n}\right)$ where $x^{\prime} \in \mathbb{R}^{n-1}$. For any unit vector $e$ such that $e \cdot \alpha>0$, we will prove that for every $t \geq 0$,

$$
u(x+t e) \geq u(x) \text { for all } x \in \mathbb{R}^{n} .
$$

This then implies that $e \cdot \nabla u \geq 0$ in $\mathbb{R}^{n}$. By continuity, this also holds for $e$ and $-e$, if $e \cdot \alpha=0$, which then implies that $e \cdot \nabla u \equiv 0$ and that $u$ depends only on $\alpha \cdot x$.

For any $t>0$, define $u^{t}(x)=u(x+t e)$. First we note that, since $e_{n}=e \cdot \alpha>0$, for $t$ large, by (1.6),

$$
u^{t}(x) \geq x_{n}+t e_{n}-C \geq x_{n}+C \geq u(x) .
$$

Hence we can define

$$
t_{0}:=\inf \{t: \forall s \geq t,(2.1) \text { holds }\}
$$

Assuming that $t_{0}>0$, we will get a contradiction. First note that $u^{t_{0}} \geq u$ by continuity. It is impossible to have $u^{t_{0}} \equiv u$, because this would imply that $u$ is $t_{0}$ periodic in the $e$ direction, which contradicts (1.6) $)(e \cdot \alpha>0$ implies that $u$ goes to infinity when $x$ goes to infinity along the $e$ direction.) Hence by the strong maximum principle we have

$$
u^{t_{0}}>u \text {. }
$$

By the definition of $t_{0}$, there exists $t_{k}<t_{0}$ such that

$$
\inf _{\mathbb{R}^{n}}\left(u^{t_{k}}-u\right)<0
$$

In particular, there exists $x_{k} \in \mathbb{R}^{n}$ such that

$$
\left(u^{t_{k}}-u\right)\left(x_{k}\right)<0 \text {. }
$$


Assume the period of $F(u)$ is $T$. By (1.6), we can take a constant $a_{k}$, which is a multiple of $T$ such that

$$
u_{k}(x):=u\left(x+x_{k}\right)-a_{k}
$$

satisfies $\left|u_{k}(0)\right| \leq T$. (2.2) and (2.3) imply respectively that

$$
\begin{gathered}
u_{k}^{t_{0}}>u_{k} . \\
\left(u_{k}^{t_{k}}-u_{k}\right)(0)<0 .
\end{gathered}
$$

Note that $u_{k}$ still satisfies (1.6) with a larger constant $2 C+T$, which is independent of $k$. By the elliptic regularity, $u_{k}$ is uniformly bounded in $C^{3}\left(B_{R}(0)\right)$ for any $R>0$. Hence we can take a subsequence of $u_{k}$ such that $u_{k}$ converges to $u_{\infty}$ in $C^{2}\left(B_{R}(0)\right)$ for any $R>0$. Letting $k \rightarrow+\infty$ in (2.4) and (2.5), we get

$$
u_{\infty}^{t_{0}} \geq u_{\infty}, u_{\infty}^{t_{0}}(0)=u_{\infty}(0) .
$$

By the strong maximum principle, $u_{\infty}^{t_{0}} \equiv u_{\infty}$. That is, $u_{\infty}$ is $t_{0}$ periodic along the direction $e$. Since $u_{\infty}$ satisfies (1.6), this is a contradiction and also finishes the proof of Theorem 1.1.

\section{Proof of Theorem 1.2}

In this section, we prove the existence of solutions satisfying estimate (1.7) in Theorem 1.2 ,

We denote $z=x+i y \in \mathbb{C}$. We also identify $z=r e^{i \theta}$ with $(x, y) \in \mathbb{R}^{2}$. Let $d \geq 2$ be a positive integer and $\varphi(x, y)=\operatorname{Re}\left(z^{d}\right)$. Denote $G$ the rotation of order $2 d$. Note that $\varphi(G z)=-\varphi(z)$.

Let $D=\left\{-\frac{\pi}{2 d}<\theta<\frac{\pi}{2 d}\right\}$ be a nodal domain of $\varphi$. For every $R>0$, take $D_{R}=B_{R}(0) \cap D$ and $u^{R}$ to be a minimizer of the functional

$$
\int_{D_{R}} \frac{1}{2}|\nabla u|^{2}+F(u),
$$

with the Dirichlet boundary condition $u=\varphi$ on $\partial D_{R}$.

First, the minimizer exists since $F(u)$ is a bounded periodic function. Second, we may assume that $u^{R} \geq 0$ in $D_{R}$ since otherwise we may replace the minimizer with $\left|u^{R}\right|$ (noting that $F$ is even and $F(|u|)=F(u)$ ). Since $F^{\prime}(u)=0$, the strong maximum principle implies that $u_{R}>0$ in $D_{R}$. Once again by the oddness of $F^{\prime}(u)$ and the fact that $F^{\prime}(0)=0$, by rotational symmetry of $\frac{2 \pi}{d}, u^{R}$ can be extended to $B_{R}(0)$ and it satisfies the equation $-\Delta u=F^{\prime}(u)$ in $B_{R}(0)$. By construction, $u^{R}$ has the same symmetry as $\varphi$, that is, $u^{R}(G z)=-u^{R}(z)$ for $z \in B_{R}(0)$ 2 In particular, the nodal domain of $u^{R}$ is the same with $\varphi$ and $\left\{u^{R}=0\right\}$ is composed by $2 d$ rays with the form $r e^{i \frac{k \pi}{2 d}}$ for $k=1,3, \cdots, 4 d-1$ and $r \in[0, R]$.

\footnotetext{
${ }^{2}$ Another method to get $u^{R}$ is to find a minimizer of $\int_{B_{R}} \frac{1}{2}|\nabla u|^{2}+F(u)$ in the invariant class $\left\{u=\varphi\right.$ on $\partial B_{R}$, and $\left.u(G z)=-u(z)\right\} \cdot u^{R}$ can be proved to satisfy $-\Delta u=F^{\prime}(u)$ in $B_{R}(0)$ by the heat flow method. Note that because $F(u)$ is even, the invariant class is positively invariant by the heat flow.
} 
For any $r \in(0, R)$, let $\varphi^{r}$ be the solution of

$$
\left\{\begin{array}{l}
\Delta \varphi^{r}=0, \text { in } B_{r}, \\
\varphi^{r}=u^{R}, \text { on } \partial B_{r} .
\end{array}\right.
$$

Since $u^{R}$ has the same symmetry as $\varphi$, by the uniqueness of the solution to the above problem, $\varphi^{r}$ has the same symmetry as $\varphi$, and $\left\{\varphi^{r}=0\right\}$ is composed by $2 d$ rays of the form $r e^{i \frac{k \pi}{2 d}}$ for $d=1,3, \cdots, 4 d-1$ and $r \in[0, r]$. This implies that $\varphi^{r}=u^{R}$ on $\partial D_{r}$ and $\varphi^{r}$ is also the harmonic extension of $u^{R}$ from $\partial D_{r}$ to $D^{r}$.

Lemma 3.1. There exists a constant $C$, independent of $r$ and $R$, such that

$$
\int_{B_{r}(0)}\left|\nabla \varphi^{r}-\nabla u^{R}\right|^{2} \leq C r^{2} .
$$

Since we expect $u^{R}$ grows like $|z|^{d}$ and $\left|\nabla u^{R}\right|$ grows like $|z|^{d-1}$ with $d \geq 2$, this estimate implies that $u^{R}$ and $\varphi^{r}$ are close to each other (after a rescaling) at large scale. Below we will use this inequality to estimate the error $u^{R}-\varphi^{r}$.

Proof. By the minimality of $u^{R}$, we have

$$
\int_{D_{r}} \frac{1}{2}\left|\nabla u^{R}\right|^{2}+F\left(u^{R}\right) \leq \int_{D_{r}} \frac{1}{2}\left|\nabla \varphi^{r}\right|^{2}+F\left(\varphi^{r}\right)
$$

which implies

$$
\int_{D_{r}}\left|\nabla u^{R}\right|^{2}-\left|\nabla \varphi^{r}\right|^{2} \leq C r^{2}
$$

since $F$ is a bounded periodic function.

On the other hand, an integration by parts using the fact that $u^{R}=\varphi^{r}$ on $\partial D_{r}$ shows that

$$
\int_{D_{r}}\left|\nabla \varphi^{r}-\nabla u^{R}\right|^{2}=\int_{D_{r}}\left|\nabla u^{R}\right|^{2}-\left|\nabla \varphi^{r}\right|^{2}
$$

Substituting the above equality into the inequality (3.2), we get (3.1).

Lemma 3.2. There exists a constant $C$, independent of $r$ and $R$, such that for all $0<r<R$,

$$
\sup _{B_{r / 2}(0)}\left|\varphi^{r}-u^{R}\right| \leq C r^{3 / 2}
$$

Proof. We will assume that $r$ is large enough. Let $\bar{u}^{r}(z):=\frac{1}{r^{d}} u^{R}(r z)$ and $\bar{\varphi}^{r}(z):=$ $\frac{1}{r^{d}} \varphi^{r}(r z)$ for $z \in B_{1}(0)$. By (3.1),

$$
\int_{B_{1}(0)}\left|\nabla \bar{\varphi}^{r}-\nabla \bar{u}^{r}\right|^{2} \leq C r^{2-2 d} .
$$

Since $\bar{u}^{r}=\bar{\varphi}^{r}$ on $\partial B_{1}(0)$, by the Poincare inequality,

$$
\int_{B_{1}(0)}\left|\bar{\varphi}^{r}-\bar{u}^{r}\right|^{2} \leq C r^{2-2 d} .
$$


Note that

$$
\left|\Delta\left(\bar{\varphi}^{r}-\bar{u}^{r}\right)\right|=\left|r^{2-d} F^{\prime}\left(r^{d} \bar{u}^{r}\right)\right| \leq C r^{2-d} .
$$

Take a $r_{0} \in(3 / 4,1)$ such that

$$
\int_{\partial B_{r_{0}}(0)}\left|\bar{\varphi}^{r}-\bar{u}^{r}\right|^{2} \leq 8 C r^{2-2 d}
$$

which is possible because of (3.3).

Take the decomposition $\bar{\varphi}^{r}-\bar{u}^{r}=h+g$, where $h$ is harmonic in $B_{r_{0}}(0)$ and $h=\bar{\varphi}^{r}-\bar{u}^{r}$ on $\partial B_{r_{0}}(0)$. By the mean value property of harmonic functions, we have

$$
\sup _{B_{5 / 8}(0)}|h| \leq C r^{1-d}
$$

Since $g=0$ on $\partial B_{r_{0}}(0)$ and

$$
|\Delta g| \leq C r^{2-d}=-\Delta\left(\frac{C r^{2-d}}{4}\left(r_{0}^{2}-|z|^{2}\right),\right.
$$

comparison principle implies

$$
\sup _{B_{5 / 8}(0)}|g| \leq C r^{2-d} .
$$

Combining these two we obtain

$$
\sup _{B_{5 / 8}(0)}\left|\bar{\varphi}^{r}-\bar{u}^{r}\right| \leq C r^{2-d} .
$$

Combining with (3.4), by elliptic estimates we see

$$
\sup _{B_{9 / 16}(0)}\left|\nabla\left(\bar{\varphi}^{r}-\bar{u}^{r}\right)\right| \leq C r^{2-d} .
$$

By (3.3),

$$
\left|\left\{\left|\bar{\varphi}^{r}-\bar{u}^{r}\right|>r^{3 / 2-d}\right\} \cap B_{9 / 16}(0)\right| \leq C r^{-1} .
$$

In particular, for any ball $B_{M r^{-1 / 2}}(x) \subset B_{9 / 16}(0)$ where $M$ is a large constant, there exists $y \in B_{M r^{-1 / 2}}(x) \cap\left\{\left|\bar{\varphi}^{r}-\bar{u}^{r}\right|<r^{3 / 2-d}\right\}$. Integrating along the segment from $y$ to $x$ and using (3.5), we get

$$
\left|\bar{\varphi}^{r}(x)-\bar{u}^{r}(x)\right| \leq C r^{3 / 2-d} \text { for any } x \in B_{1 / 2}(0) .
$$

Rescaling back we can finish the proof.

Lemma 3.3. There exists a constant $C$, independent of $r$ and $R$, such that for any $r \in(0, R / 2)$,

$$
\sup _{B_{r / 2}(0)}\left|\nabla^{2} \varphi^{r}-\nabla^{2} \varphi^{2 r}\right| \leq \frac{C}{r} .
$$


Proof. By (3.1) we get

$$
\int_{B_{r}(0)}\left|\nabla \varphi^{r}-\nabla \varphi^{2 r}\right|^{2} \leq C r^{2}
$$

Since both $\varphi^{r}$ and $\varphi^{2 r}$ are harmonic, by interior gradient estimates we obtain the claim.

Lemma 3.4. For any $r \in(0, R)$,

$$
\sup _{B_{r / 2}(0)}\left|\varphi-u^{R}\right| \leq C r^{3 / 2}
$$

Proof. Take an $i_{0}$ such that $R / 2<2^{i_{0}} r \leq R$. Checking the proof of the previous lemma we see

$$
\sup _{B_{2^{i_{0}-1_{r}}(0)}}\left|\nabla^{2} \varphi^{2^{i_{0} r}}-\nabla^{2} \varphi\right| \leq \frac{C}{2^{i_{0} r}} .
$$

Adding this and (3.6) from $i=1$ to $i=i_{0}$ we get

$$
\sup _{B_{r / 2}(0)}\left|\nabla^{2} \varphi^{r}-\nabla^{2} \varphi\right| \leq \frac{C}{r} .
$$

Since for each $r, \varphi^{r}$ has the same symmetry as $\varphi$ and it is harmonic (recall that the degree of $\varphi, d \geq 2$ ), we have

$$
\varphi^{r}(0)=\varphi(0)=0, \nabla \varphi^{r}(0)=\nabla \varphi(0)=0 .
$$

Integrating (3.7) twice we obtain,

$$
\sup _{B_{r / 2}(0)}\left|\varphi^{r}-\varphi\right| \leq C r
$$

This combined with Lemma 3.2 implies the required claim.

A direct corollary of this lemma is the uniform boundedness of $u^{R}$ on any compact set. Hence we can take the limit $u_{\infty}:=\lim _{R \rightarrow+\infty} u^{R}$ which is a solution of (1.5) on the entire $\mathbb{R}^{2}$, enjoying the same symmetry as $\varphi,\left\{u_{\infty}>0\right\}=\{\varphi>0\}$, and satisfies

$$
\left|u_{\infty}(x, y)-\varphi(x, y)\right| \leq C(|x|+|y|)^{3 / 2} .
$$

In particular, $u_{\infty}$ is unbounded and grows at least quadratically.

This proves Theorem 1.2 .

Remark 3.5. By [1], there exists a second solution u of (1.5) satisfying the symmetry $u(G z)=-u(z)$, which is bounded in $\mathbb{R}^{2}$. For example, if $F(u)=1+\cos u$, we can construct a solution such that $-\pi<u<\pi$ in $\mathbb{R}^{2}$. In fact, in this case, if we modify $F(u)$ outside $[-\pi, \pi]$ to get a standard double-well potential, it becomes exactly the problem studied in [1, 3]. The bounded solution produced by this method takes values in $(-\pi, \pi)$ and it is still the solution of the original problem (1.5). 


\section{PROOF OF THE IMPROVEMENT ESTIMATE (1.8)}

Let $u$ be the solution constructed in the previous section. Written in the exponential polar coordinate $(r, \theta)=\left(e^{t}, \theta\right)$, u satisfies

$$
\partial_{t}^{2} u+\partial_{\theta}^{2} u+e^{2 t} f(u)=0 .
$$

Let $v(t, \theta)=e^{-d t} u(t, \theta)$. Then $v(t, \theta)$ satisfies

$$
\partial_{t}^{2} v+2 d \partial_{t} v+d^{2} v+\partial_{\theta}^{2} v+e^{(2-d) t} f\left(e^{d t} v\right)=0 .
$$

By the error bound established in the previous section, for $t \geq 0$,

$$
|v(t, \theta)-\cos (d \theta)| \leq C e^{(3 / 2-d) t} .
$$

By interior gradient estimates, for any $\varepsilon>0$ there exists a constant $C$ such that for any ball $B_{1}(t, \theta) \subset \mathbb{R} \times \mathbb{S}^{1}$ (with respect to the product metric on $\mathbb{R} \times \mathbb{S}^{1}$ ) and $u \in C^{2}\left(B_{1}(t, \theta)\right)$,

$$
\sup _{B_{1 / 2}(t, \theta)}\left|\partial_{\theta} u\right|+\left|\partial_{t} u\right| \leq \varepsilon \sup _{B_{1}(t, \theta)}\left|\partial_{t}^{2} u+\partial_{\theta}^{2} u\right|+\frac{C}{\varepsilon} \sup _{B_{1}(t, \theta)}|u| .
$$

Since $\left|e^{(2-d) t} f\left(e^{d t} v\right)\right| \leq C e^{(2-d) t}$, applying (4.3) to $v-\cos (d \theta)$ with $\varepsilon=e^{-\frac{t}{4}}$ we get a constant $C$ such that for all $t \geq 0$,

$$
\left|\partial_{\theta}(v(t, \theta)-\cos (d \theta))\right|+\left|\partial_{t} v(t, \theta)\right| \leq C e^{\left(\frac{7}{4}-d\right) t}
$$

Differentiating (4.1) in $t$ we get

$$
\partial_{t}^{2} \partial_{t} v+2 d \partial_{t} \partial_{t} v+d^{2} \partial_{t} v+\partial_{\theta}^{2} \partial_{t} v+e^{2 t} f^{\prime}\left(e^{d t} v\right) \partial_{t} v=0
$$

By the bound on $\partial_{t} v$, we have $\left|e^{2 t} f^{\prime}\left(e^{d t} v\right) \partial_{t} v\right| \leq C e^{\left(\frac{7}{4}+2-d\right) t}$. By taking $\varepsilon=e^{-t}$ in (4.3) we obtain

$$
\left|\partial_{\theta} \partial_{t} v(t, \theta)\right|+\left|\partial_{t}^{2} v(t, \theta)\right| \leq C e^{\left(\frac{7}{4}+1-d\right) t} .
$$

Substituting this and (4.2), (4.4) into (4.1), we get

$$
\left|\partial_{\theta}^{2}(v(t, \theta)-\cos (d \theta))\right| \leq C e^{\left(\frac{7}{4}+1-d\right) t}
$$

If $d \geq 3$, this gives the exponential convergence of $v$ to $\cos (d \theta)$ in $C^{2}\left(\mathbb{S}^{1}\right)$.

Below we assume that $d \geq 3$.

Let $v(t, \theta)=\sum_{j \geq 0} c_{j}(t) \cos (j \theta)$ be the Fourier decomposition of $v(t, \cdot)$. Note that because $v$ is even in $\theta$, there are only terms $\cos (j \theta)$ appearing in this decomposition. Moreover, by our construction,

$$
\sum_{j \geq 0} c_{j}(t) \cos \left(j \theta+\frac{j \pi}{d}\right)=v\left(t, \theta+\frac{\pi}{d}\right)=-v(t, \theta)=-\sum_{j \geq 0} c_{j}(t) \cos (j \theta),
$$

so $c_{j}(t)=0$ if there is no nonnegative integer $k$ such that $j=(2 k+1) d$. In particular,

$$
c_{j}(t) \equiv 0 \text { for } j<d \text {. }
$$

Hence below we concentrate on those $c_{j}(t)$ with $j=d$ and $j \geq 3 d$. 
Multiplying (4.1) by $\cos (j \theta)$ and integrating, we get the equation for $c_{j}(t)$

$$
\partial_{t}^{2} c_{j}+2 d \partial_{t} c_{j}+\left(d^{2}-j^{2}\right) c_{j}+e^{(2-d) t}\left(\int_{0}^{2 \pi} f\left(e^{d t} v\right) \cos (j \theta) d \theta\right)=0 .
$$

Denote $g_{j}(t)=e^{(2-d) t}\left(\int_{0}^{2 \pi} f\left(e^{d t} v\right) \cos (j \theta) d \theta\right)$. Since $\cos (d \theta)$ has only non-degenerate critical points and $v(t, \theta) \rightarrow \cos (d \theta)$ in $C^{2}\left(\mathbb{S}^{1}\right)$ as $t \rightarrow+\infty$ (cf. (4.4) and (4.5)), for $t$ large, $v(t, \cdot)$ has only non-degenerate critical points. By the oscillatory integral estimate ( $\left[\right.$ Section 8.1, [11]]) we get a constant $C_{j}$ such that

$$
\int_{0}^{2 \pi} f\left(e^{d t} v\right) \cos (j \theta) d \theta=O\left(e^{-\frac{d t}{2}}\right), \quad\left|g_{j}(t)\right| \leq C_{j} e^{\left(2-\frac{3 d}{2}\right) t}
$$

For $t \geq 0$, we have the representation formula

$$
c_{j}(t)=A_{j} e^{-(d+j) t}+B_{j} e^{-(d-j) t}+e^{-(d-j) t} \int_{t}^{+\infty} e^{(d-j) s} \int_{0}^{s} e^{(d+j)(\tau-s)} g_{j}(\tau) d \tau d s .
$$

Substituting (4.6) into this and integrating directly, we see the last integral is bounded by $\frac{C_{j}}{j^{2}} e^{\left(2-\frac{3 d}{2}\right) t}$. In particular, for $j=d$,

$$
\left|c_{d}(t)-B_{d}\right| \leq C e^{\left(2-\frac{3 d}{2}\right) t} .
$$

Here, by (4.2), $B_{d}=1$.

It remains to estimate $v^{\|}:=v-c_{d}(t) \cos (d \theta)$. First note that for $j>d,\left|c_{j}(t)\right| \leq$ $C e^{(3 / 2-d) t}$ by (4.2). Hence we must have $B_{j}=0$. Next we have

Lemma 4.1. For $t$ large, when measured in $L^{\infty}\left(\mathbb{S}^{1}\right)$,

$$
v^{b}:=\sum_{j>d} e^{-(d-j) t} \int_{t}^{+\infty} e^{(d-j) s} \int_{0}^{s} e^{(d+j)(\tau-s)} g_{j}(\tau) \cos (j \theta) d \tau d s=O\left(e^{\left(2-\frac{3 d}{2}\right) t}\right) .
$$

Proof. Direct calculations give, for $t+\tau<2 s$,

$\sum_{j>d} e^{-(d-j) t} e^{(d-j) s} e^{(d+j)(\tau-s)} \cos (j \theta)=e^{t-(2 d+2) s+(2 d+1) \tau} \frac{\cos (d+1) \theta-e^{t+\tau-2 s} \cos d \theta}{1-2 e^{t+\tau-2 s} \cos \theta+e^{2(t+\tau-2 s)}}$.

Using this kernel, $v^{b}$ can be written as

$v^{b}(t, \theta)=\int_{t}^{+\infty} \int_{0}^{s} \int_{0}^{2 \pi} e^{t-(2 d+2) s+(2 d+1) \tau} \frac{\cos (d+1) \theta-e^{t+\tau-2 s} \cos d \theta}{1-2 e^{t+\tau-2 s} \cos \theta+e^{2(t+\tau-2 s)}} g(\tau, \theta) d \theta d \tau d s$,

where $g(\tau, \theta)=e^{(2-d) \tau} f\left(e^{d \tau} v(\tau, \theta)\right)$.

Note that

$$
\frac{\cos (d+1) \theta-e^{t+\tau-2 s} \cos d \theta}{1-2 e^{t+\tau-2 s} \cos \theta+e^{2(t+\tau-2 s)}}
$$

is uniformly bounded in $C^{3}\left(\mathbb{S}^{1}\right)$ when $t+\tau-2 s \leq 0$. Hence by the oscillatory integral estimate $([1])$,

$$
\int_{0}^{2 \pi} \frac{\cos (d+1) \theta-e^{t+\tau-2 s} \cos d \theta}{1-2 e^{t+\tau-2 s} \cos \theta+e^{2(t+\tau-2 s)}} g(\tau, \theta) d \theta=O\left(e^{\left(2-\frac{3 d}{2}\right) \tau}\right) .
$$


Substituting this into the above representation formula of $v^{b}$ we finish the proof.

Lemma 4.2. For $t$ large, when measured in $L^{\infty}\left(\mathbb{S}^{1}\right)$,

$$
v^{\sharp}:=v^{\|}-v^{b}=O\left(e^{-\frac{d-1+\sqrt{17 d^{2}-2 d+1}}{2} t}\right) .
$$

Proof. Direct calculations show that

$$
\left(\partial_{t}^{2}+2 d \partial_{t}+\partial_{\theta}^{2}+d^{2}\right) v^{b}+e^{(2-d) t} f\left(e^{d t} v(t, \theta)\right)=0 .
$$

Hence

$$
\left(\partial_{t}^{2}+2 d \partial_{t}+\partial_{\theta}^{2}+d^{2}\right) v^{\sharp}=0 .
$$

Multiplying by $v^{\sharp}$ and integrating on $\mathbb{S}^{1}$, we get

$$
\frac{d^{2}}{d t^{2}} \int_{0}^{2 \pi}\left(v^{\sharp}\right)^{2} d \theta+(2 d-2) \frac{d}{d t} \int_{0}^{2 \pi}\left(v^{\sharp}\right)^{2} d \theta+2 d^{2} \int_{0}^{2 \pi}\left(v^{\sharp}\right)^{2} d \theta-2 \int_{0}^{2 \pi}\left(\partial_{\theta} v^{\sharp}\right)^{2} d \theta=0 .
$$

By our construction, for any $t \geq 0, v^{\sharp}$ is orthogonal to $\cos (j \theta), \sin (j \theta)$ for every $|j| \leq 3 d-1$. Hence

$$
\int_{0}^{2 \pi}\left(\partial_{\theta} v^{\sharp}\right)^{2} d \theta \geq(3 d)^{2} \int_{0}^{2 \pi}\left(v^{\sharp}\right)^{2} d \theta,
$$

and

$$
L \int_{0}^{2 \pi}\left(v^{\sharp}\right)^{2} d \theta:=\frac{d^{2}}{d t^{2}} \int_{0}^{2 \pi}\left(v^{\sharp}\right)^{2} d \theta+(2 d-2) \frac{d}{d t} \int_{0}^{2 \pi}\left(v^{\sharp}\right)^{2} d \theta-16 d^{2} \int_{0}^{2 \pi}\left(v^{\sharp}\right)^{2} d \theta \geq 0 .
$$

By (4.2), (4.8) and the previous lemma, we have the decay estimate

$$
\int_{0}^{2 \pi} v^{\sharp}(t, \theta)^{2} d \theta \leq C e^{(3-2 d) t} .
$$

Let $\left(\int_{0}^{2 \pi}\left(v^{\sharp}(0, \theta)^{2} d \theta\right) e^{-\left(d-1+\sqrt{17 d^{2}-2 d+1}\right) t}\right.$ be a solution of $L h=0$ which has the same boundary value at $t=0$ and $t=+\infty$. By the comparison principle we get for any $t \geq 0$

$$
\int_{0}^{2 \pi} v^{\sharp}(t, \theta)^{2} d \theta \leq\left(\int_{0}^{2 \pi} v^{\sharp}(0)^{2} d \theta\right) e^{-\left(d-1+\sqrt{17 d^{2}-2 d+1}\right) t} .
$$

Then by applying standard elliptic estimates to $v^{\sharp}$ we get its $L^{\infty}\left(\mathbb{S}^{1}\right)$ bound.

For $d \geq 2$,

$$
\frac{d-1+\sqrt{17 d^{2}-2 d+1}}{2} \geq \frac{3 d}{2}-2
$$

Putting the above estimates together we see for every $d \geq 3$,

$$
\sup |v(t, \theta)-\cos (d \theta)| \leq C e^{-\left(\frac{3 d}{2}-2\right) t} .
$$

Coming back to $u$, we get a constant $C$ such that for all $z \in \mathbb{C}$

$$
|u(z)-\varphi(z)| \leq C(1+|z|)^{-\left(\frac{d}{2}-2\right)}
$$

which proves (1.8). 


\section{REFERENCES}

[1] F. Alessio, A. Calamai and P. Montecchiari, Saddle-type solutions for a class of semilinear elliptic equations. Adv. Differential Equations 12 (2007), no. 4, 361-380.

[2] V. Bangert, On minimal laminations of the torus, AIHP Analyse Nonlineaire 6(1989), 95-138.

[3] H. Dang, P. C. Fife and L. A. Peletier, Saddle solutions of the bistable diffusion equation, Z. Angew. Math. Phys. 43 (1992), no. 6, 984-998.

[4] M.del Pino, M.Kowalczyk, F.Pacard and J.Wei, Multiple-end solutions to the AllenCahn equation in $\mathbb{R}^{2}$, J. Funct. Anal. 258(2010), no.2, 458-503.

[5] A. Farina and E. Valdinoci, Some results on minimizers and stable solutioons of a variational problem, Ergodic Theory and Dynamical Systems 32(2012), 1302-1312.

[6] J. Moser, Minimal solutions of variational problems on a torus, AIHP Analyse Nonlineaire 3(1986), 229-272.

[7] P. H. Rabinowitz, Single and multitransition solutions for a family of semilinear elliptic PDEs, Milan J. Math. 179(2011), 113-127.

[8] P. H. Rabinowitz and E. Stredulinsky, Mixed states for an Allen-Cahn type equation, Comm. Pure Appl. Math. 56(2003), 1078-1134.

[9] P. H. Rabinowitz and E. Stredulinsky, Mixed states for an Allen-Cahn type equation, II, Cal. Var. Partial Differential Equations 21(2004), 157-207.

[10] P. H. Rabinowitz and E. Stredulinsky, Extensions of Moser-Bangert Theory: Locally Minimal Solutions, Progress in Nonlinear Differential Equations and Their Applications Volume 81, Birkhauser.

[11] E. M. Stein and T. S. Murphy, Harmonic Analysis: Real-Variable Methods, Orthogonality, and Oscillatory Integrals, Princeton University Press, 1993

Wuhan Institute of Physics and Mathematics, The Chinese Academy of Sciences, Wuhan 430071, China. EMail: WANGKelei@WIPM.AC.CN

Department of Mathematics, The Chinese University of Hong Kong, Shatin, Hong Kong. EMAIL: WEI@MATH.CUHK.EDU.HK 